\title{
Multiscale Systems Biology: A Special Issue Devoted to Understanding Biology and Medicine Across Multiple Scales
}

\author{
Michael R. KING ${ }^{1}$ and Scott L. Diamond ${ }^{2}$ \\ ${ }^{1}$ Department of Biomedical Engineering, Cornell University, Ithaca, NY, USA; and ${ }^{2}$ Department of Chemical and Biomolecular \\ Engineering, University of Pennsylvania, Philadelphia, PA, USA
}

The central goal of Systems Biology is to understand and quantitatively predict biological phenomena at a global level, where biological functions result from complex mechanisms that occur across diverse scales, from the atomic and molecular to the organelle and cellular level, and then extended to the tissue, organism, and population level. In bioengineering applications, unique challenges include: (1) the regulatory and kinetic complexity of gene and metabolic and signaling pathways, (2) generation or response to physical forces, and (3) complex transport physics.

The task of identifying disease mechanism, disease severity, or response to therapy (i.e., phenotype) remains difficult if the only data set available were a patient's genome sequence. Even with increasingly powerful supercomputers, brute force approaches at a single scale often prove inadequate to tackle these multiscale problems, especially when physical or thermodynamic criteria are also imposed.

With potentially many scales and many variables associated with problems of interest, the need for systematic approaches to modeling multiscale systems motivates this Special Issue. This collection of 16 papers from leading laboratories highlights these advances in distinct medical arenas.

Modeling and the discovery of emergent properties, for instance cellular processes arising from cell signaling networks, are best addressed by following a systems biology approach. One important theme in multiscale modeling is coarse graining, where some of the fine detail of low-level models has been smoothed over or averaged out. Another important concept in multiscale modeling is linking between scales; that is, how one reconciles coupling fine-scale and coarser-

Address correspondence to Michael R. King, Department of Biomedical Engineering, Cornell University, Ithaca, NY, USA and Scott L. Diamond, Department of Chemical and Biomolecular Engineering, University of Pennsylvania, Philadelphia, PA, USA. Electronic mail: mike.king@cornell.edu, sld@seas.upenn.edu grained models in an accurate, predictive, and unambiguous way. In this Special Issue, a variety of approaches and methodologies are presented for the use of multiscale computational models to study and predict the behavior of complex systems.

The goal of this special issue is to highlight advances in the field of biomedical engineering that tackle multiscale problems. The field has advanced rapidly and the National Institutes of Health has supported several initiatives related to Multiscale Systems Biology to address clinical needs, including the establishment of a Multiscale Modeling Consortium. ${ }^{1}$ The Annals of Biomedical Engineering is an ideal platform to communi-

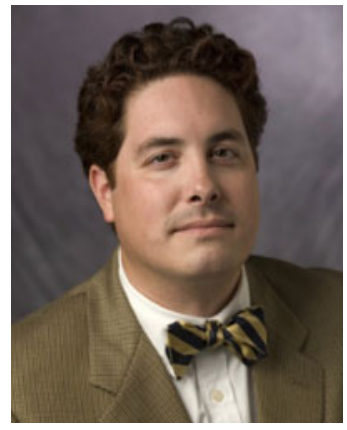

Michael R. King.

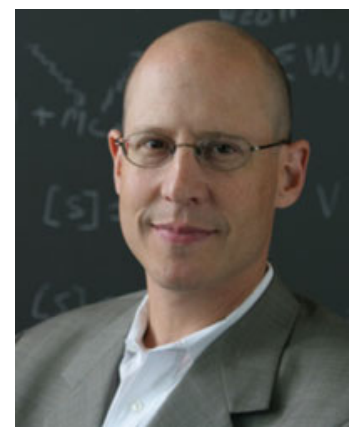

Scott L. Diamond. cate these advances and state of the art approaches. This special issue highlights bioengineering research that emphasizes molecular-cellular-tissue scale simulation and measurement, for instance when combined with aspects of high throughput experiments, physical biology, or mechanobiology. Many of the papers in this issue describe computational biology approaches that are combined with experimental validation or confirmation.

The articles featured in this special issue address several different physiological systems and cellular phenomena. Signal transduction at the molecular level is considered in papers by Radakrishnan and colleagues, and by Wilson and colleagues. Janes and colleagues describe multiscale models of cell signaling. Calvetti and colleagues discuss the metabolism of 
neural cells. Two papers on the function of blood platelets and thrombosis are contributed by the King and Diamond groups. Different multiscale studies of the function of the heart are provided by Carlson, Beard et al., by Bassingthwaighte and coworkers, as well as a study of coronary circulation by Smith et al. Vodovotz et al. describe models of sepsis and inflammation. Parthak, Popel et al. present a multiscale model and imaging of tumor angiogenesis, and $\mathrm{Fu}$ and colleagues describe macrovascular transport and tumor cell adhesion. Finally, in the orthopaedic biomechanics field, Erdemir et al. describe multiscale mechanics in cartilage, and Webster, Muller et al. discuss the systems biology of bone.

We hope that you will find these articles exciting, informative and timely in highlighting current research in Multiscale Systems Biology.

\section{REFERENCE}

${ }^{1}$ http://www.imagwiki.nibib.nih.gov/mediawiki/index.php? title $=$ Multiscale_Systems_Biology_Working_Group. 